\title{
Stressed-Metal Probes for Atomic Force Microscopy on Biological Samples
}

\author{
T. Hantschel,* E.M. Chow, ${ }^{*}$ and L. Wong*
}

Palo Alto Research Center, 3333 Coyote Hill Road, Palo Alto, CA 94304, California, USA

Common probes for atomic force microscopy (AFM) are based on straight silicon or silicon nitride cantilevers with integrated micrometer-sized tips [1,2]. Metal and diamond probes are often used for electrical AFM measurements [3,4]. Despite the high resolution and availability of such probes, their low tip height does not allow for samples with high topography. Therefore, a configuration called stressed-metal probe has recently been developed which uses the end of a bent cantilever as a tip in AFM measurements [5]. Hundreds of micrometer tall tips can be made in this way. Probes with smaller tip heights of tens of micrometers are an alternative to common probes for topography measurements.

During the past few years, AFM has also become a powerful tool for the characterization of biological samples [6] such as DNA and living cells. The AFM imaging of samples in an aqueous medium has received special attention because it allows to the study of cells in their natural environment and provides higher resolution than optical microscopy. Fluid cells are however required for such measurements, which results in a more complicated setup compared to topography measurements in air. Therefore, we have developed an alternative approach whereby a stressedmetal probe itself can serve as a fluid cell. This paper presents first results on using stressed-metal probes for imaging biological samples and demonstrates its use for imaging in aqueous medium.

Fig. 1a shows a schematic of how a stressed-metal probe is used to scan on dry biological samples. The bent cantilever is fixed to a transparent probe chip and its tapered end is used as a probe tip in AFM. The laser shines through the probe chip. Fig. $1 \mathrm{~b}$ illustrates how a stressed-metal probe can be transformed into a simple fluid cell. As can be seen, the fluid is enclosed between the transparent chip and the substrate surface. Probes with tip heights between 40 and $150 \mu \mathrm{m}$ were used in the experiments. Fig. 2 shows a stressed NiZr probe imaged by scanning electron microscopy (SEM). This particular probe has four cantilevers on quartz which are 400, 300, 200 and $100 \mu \mathrm{m}$ long. Fig. $3 \mathrm{a}$ is an example where such a probe was used to image a mica surface coated with linear DNA molecules in an air environment. The image was taken in non-contact mode. Fig. $3 \mathrm{~b}$ demonstrates the use of a stressed-metal probe as a simple fluid cell. In this case, a calibration sample with $80 \mathrm{~nm}$ high silicon nitride structures was scanned in contact mode. Current work includes imaging biological samples in an aqueous medium.

\section{References}

[1] O. Wolter et al., J. Vac. Sci. Technol. B 9 (1991) 1353.

[2] T. R. Albrecht et al., J. Vac. Sci. Technol. A 8 (1990) 3386.

[3] T. Hantschel et al., Proc. SPIE 4175 (2000) 50.

[4] Ph. Niedermann et al., Appl. Phys. A: Mater. Sci. Process. 66 (1998) S31.

[5] T. Hantschel et al., Appl. Phys. Lett. 81(16) (2002) 3070.

[6] H.G. Hansma et al., Biophys. J. 70 (1996) 1933. 

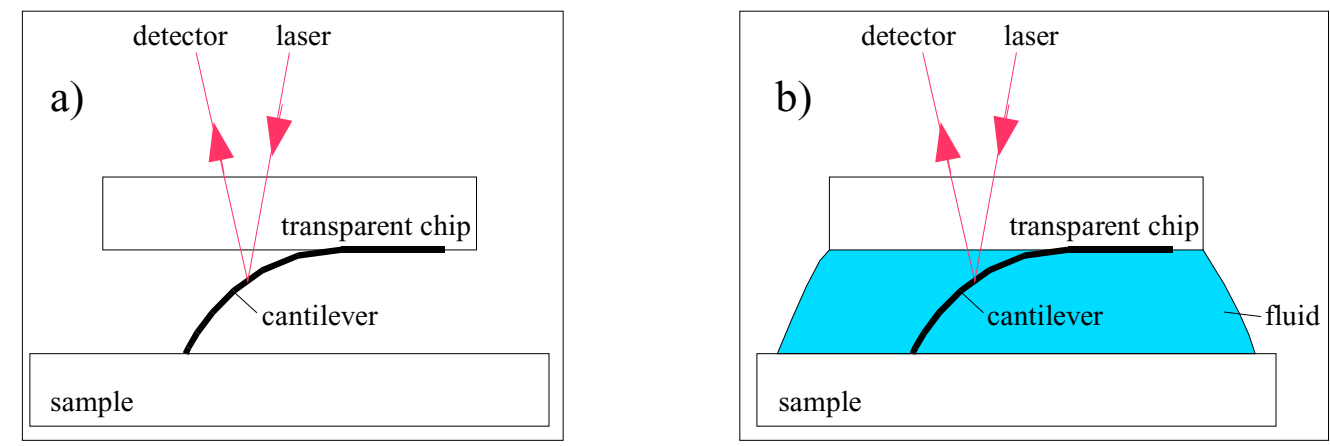

FIG. 1. Concept of StressedMetal AFM probe: a) use on dry surface, b) use as simple fluid cell.

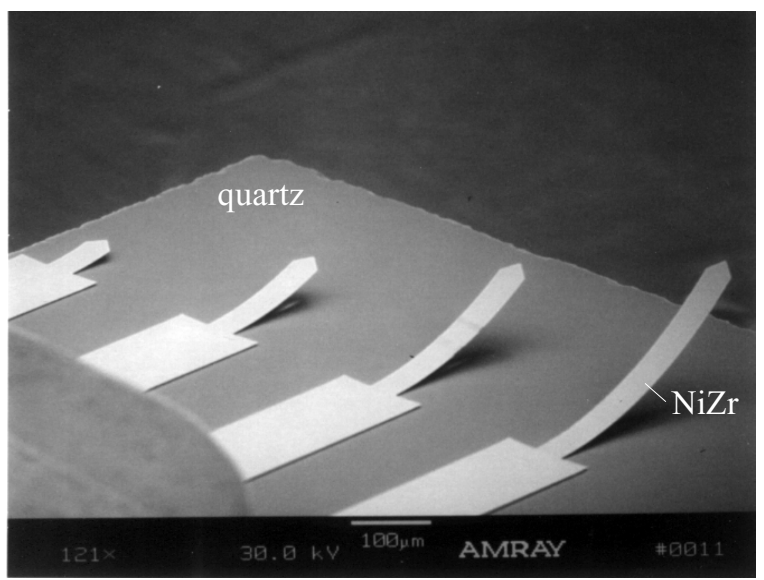

FIG. 2. StressedMetal NiZr probe with four cantilevers imaged by SEM.
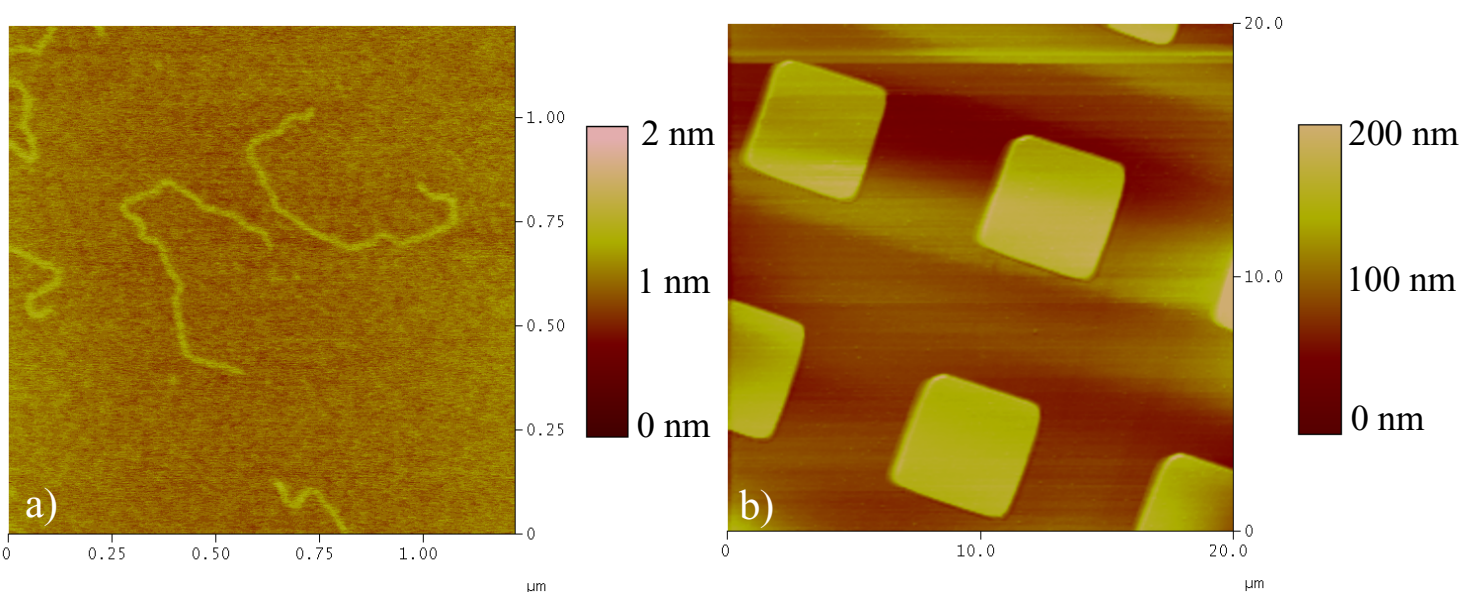

FIG. 3: AFM images taken with NiZr probe: a) $1.2 \times 1.2 \mu \mathrm{m}^{2}$ non-contact mode scan of DNA molecules on mica surface in air environment, b) $20 \times 20 \mu \mathrm{m}^{2}$ contact mode scan of silicon nitride structures on silicon in aqueous environment. 